\title{
Modelling and Predictive Control of Inverted Pendulum
}

\author{
Petr Chalupa \\ Vladimír Bobál \\ Department of Process Control \\ Tomas Bata University in Zlin \\ Mostni 5139, 760 01, Zlin, Czech Republic \\ E-mail: chalupa@fai.utb.cz
}

\section{KEYWORDS}

Modelling, State space model, Inverted Pendulum, Predictive control.

\begin{abstract}
The paper is focused on creating a model of Inverted pendulum system and subsequent usage of this model to design a predictive controller of inverted pendulum system. The model is obtained on base of mathematical physical analysis of the system. Unknown parameters of the model are obtained from real-time experiments on the PS600 Inverted pendulum system. The model is designed in MATLAB/Simulink environment. The model was created with respect to most nonlinearities contained in the system. Nonlinearities are caused by fundamental principles of the system and by friction between individual parts of the system. Thus, the model is highly non-linear and therefore linearization around working point was performed and continuous linearized model was calculated as well as its discrete version. The discrete linear model was used to design predictive controller which was also verified by real time experiments.
\end{abstract}

\section{INTRODUCTION}

Most of current control algorithms are based on a model of a controlled plant (Bobál et al. 2005). It is obvious that some information about controlled plant is required to be able to control output of the plant or to investigate its properties and behaviour. Two basic approaches of obtaining plant model exist: the black box approach and the mathematical-physical analysis of the plant.

The black box approach to the modelling (Liu, 2001) is based on analysis of input and output signals of the plant. The main advantages of this approach lies in the possibility of usage the same identification algorithm for wide set of different controlled plants. Also, the knowledge of physical principle of controlled plant and solution of possibly complicated set of mathematical equation is not required. On the other hand, model obtained by black box approach is generally valid only for signals it was calculated from. For example, if step response was used to obtain the model, this model should not be used for analysis of the system behaviour when high frequency changes of input signals are applies. The mathematical-physical analysis of the plant and following derivation of the relations between plant inputs and outputs provides general model which can be valid for whole range of plant inputs and states. Contrary, there is usually a lot of unknown constants and relations in the model description when performing mathematic-physical analysis.

The second method, mathematical-physical analysis, is used in this paper. The goal of the work was to obtain a mathematical model of the PS600 Inverted Pendulum System (Amira, 2000), to design the model in MATLAB-Simulink environment and use this model for a design of model predictive controller. The PS600 laboratory equipment was developed by Amira Gmbh, Duisburg, Germany and serves as a real-time model of unstable, highly nonlinear system.

When the model of the controlled system is known the problem of selecting an appropriate control synthesis arises. Many successful control techniques have been developed in past decades. One of them is model predictive control (MPC) (Camacho and Bordons, 2004). Contrary to most other approaches, MPC uses not only current and previous values of control circuit signals but also future values of reference signal. Future course of reference signal is known in many applications and thus can be used in controller synthesis.

The scheme of a simple control circuit with self-tuning predictive controller is shown in Figure. 1. Note that the reference signal is marked as $\mathrm{w}(\mathrm{t})$, which means that the course of reference signal is sent to the controller, not only the current value $\mathrm{w}(\mathrm{k})$.

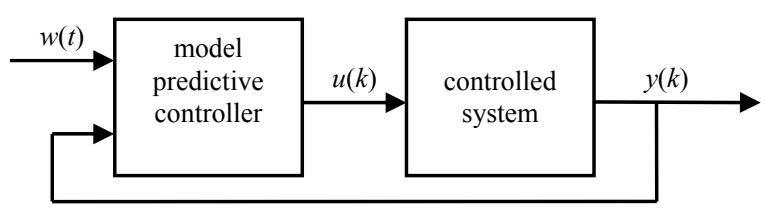

Figure 1: Control circuit with model predictive controller

\section{INVERTED PENDULUM SYSTEM}

The PS600 inverted pendulum system is shown in figure 2 . The main parts of the system are cart driven by 
servo amplifier and the pendulum rod attached to the cart.

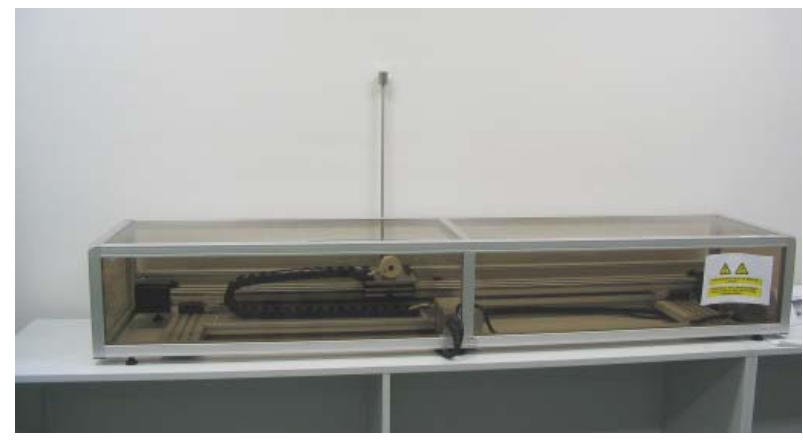

Figure 2: Photo of PS600 Inverted Pendulum system

A simplified scheme of the inverted pendulum system is shown in figure 3. This scheme was used for mathematical physical analysis of the system.

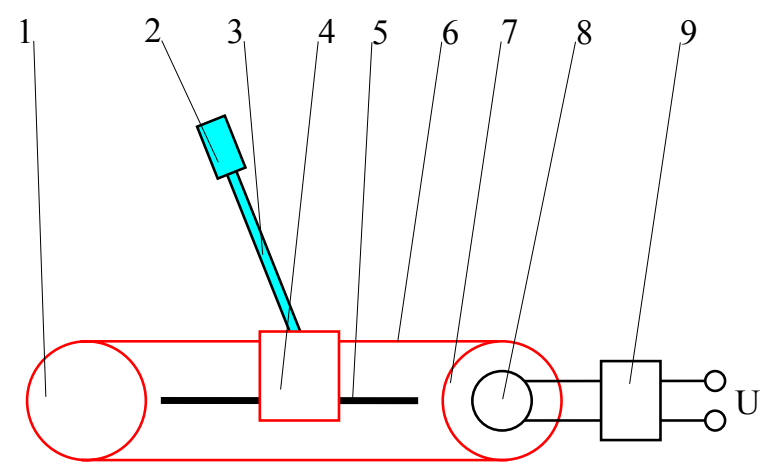

$$
\begin{array}{ll}
1 \text { - guide roll } & 6 \text { - transmission belt } \\
2 \text { - pendulum weight } & 7 \text {-drive wheel } \\
3 \text { - pendulum rod } & 8 \text { - DC motor } \\
4 \text { - cart } & 9 \text { - servo amplifier } \\
5 \text { - guiding bar } &
\end{array}
$$

Figure 3: Scheme of inverted pendulum

The PS600 Inverted Pendulum is designed as a system with one input and two measured outputs - SITO (single input two outputs). The input of the system is a control voltage of the servo amplifier $(U)$ and the outputs are cart position and angle of pendulum rod. Both outputs are measured by incremental encoders (Amira, 2000).

Systems allows different control objectives with various difficultness of control design. The most common cases are:

- control of cart position with pendulum acting as a disturbance

- control of cart position with stabilization of the pendulum in the stable equilibrium position (ie. pendulum underneath the guiding bar)

- stabilization of the pendulum in the upright position
- control of the cart position with pendulum in upright position.

\section{MATHEMATICAL MODEL}

Forces and moments acting in the system were analysed using figure 4 where $\varphi$ represents the angle of pendulum rod, $M_{0}$ and $M_{1}$ stands for the weight of the cart and pendulum respectively, $l_{S}$ is a distance between centre of gravity of the pendulum and the centre of rotation of the pendulum and $g$ is the gravity acceleration constant. Symbol $F$ represents the force produced by the DC motor.

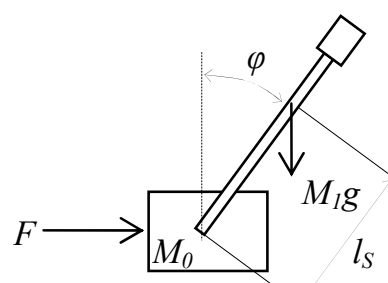

Figure 4: Analysis of inverted pendulum

It is obvious that the position and dynamics of the pendulum affects the cart. This affect is described by a force which can be divided into horizontal and vertical components. The horizontal component of the force is:

$$
H=M_{1} \frac{\partial^{2}}{\partial t^{2}}\left(r+l_{S} \sin \varphi\right)
$$

where $r$ represents position of the cart. The vertical component is:

$$
V=M_{1} \frac{\partial^{2}}{\partial t^{2}}\left(l_{S} \cos \varphi\right)
$$

The motion equation of the cart can be written as follows:

$$
M_{0} \frac{\partial^{2} r}{\partial t^{2}}=F-H-F_{r} \frac{\partial r}{\partial t}
$$

where $F_{r}$ represents constant of a velocity proportional friction of the cart. According to the angular momentum conservation law, the rotary motion of the rod about its centre is described as:

$$
\Theta_{S} \frac{\partial^{2} \varphi}{\partial t^{2}}=V l_{S} \sin \varphi-H l_{S} \cos \varphi-C \frac{\partial \varphi}{\partial t}
$$

where $\Theta_{S}$ represents the inertia moment of the pendulum rod with respect to the centre of gravity and $C$ denotes the friction constant of the pendulum. 
Substituting equations (1) and (2) into equations (3) and (4) leads to the description of behaviour of the system by set of two nonlinear differential equations:

$$
\begin{gathered}
M r^{\prime \prime}+F_{r} r^{\prime}+M_{1} l_{S} \varphi^{\prime \prime} \cos \varphi-M_{1} l_{S}\left(\varphi^{\prime}\right)^{2} \sin \varphi=F \\
\Theta \varphi^{\prime \prime}+C \varphi^{\prime}-M_{1} l_{S} g \sin \varphi+M_{1} l_{S} r^{\prime \prime} \cos \varphi=0
\end{gathered}
$$

where following abbreviations were used:

$$
\begin{aligned}
& \Theta=\Theta_{S}+M_{1} l_{S}^{2} \\
& M=M_{0}+M_{1}
\end{aligned}
$$

The input of the system is represented by a force F. This force is proportional to the control voltage of servo amplifier.

The model described by equations (5) and (6) was created in the MATLAB/Simulink environment as a standalone block. This approach can be used in simulations or as a part of rapid prototyping of a controller. The Simulink scheme is shown in figure 5.

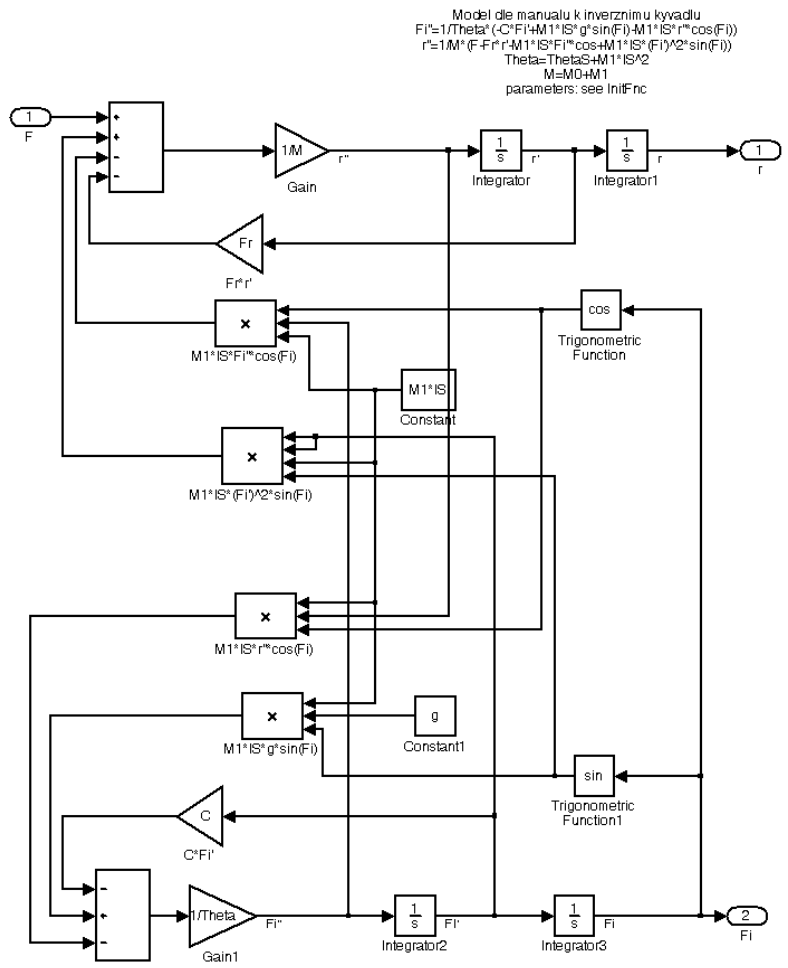

Figure 5: Simulink scheme of the nonlinear model

\section{State description of the model}

Equations (5) and (6) describe behaviour of the inverted pendulum. To obtain state space description of the system, following state vector was introduced:

$$
\boldsymbol{x}=\left[\begin{array}{c}
r \\
r^{\prime} \\
\varphi \\
\varphi^{\prime}
\end{array}\right]=\left[\begin{array}{c}
x_{1} \\
x_{2} \\
x_{3} \\
x_{4}
\end{array}\right]
$$

Then, the dynamics of the system can be described by a vector function

$$
\boldsymbol{x}^{\prime}=\left[\begin{array}{c}
x_{1}^{\prime} \\
x_{2}^{\prime} \\
x_{3}^{\prime} \\
x_{4}^{\prime}
\end{array}\right]=\boldsymbol{f}(\boldsymbol{x}, F)=\left[\begin{array}{c}
x_{2} \\
f_{2}(\boldsymbol{x}, F) \\
x_{4} \\
f_{4}(\boldsymbol{x}, F)
\end{array}\right]
$$

Functions $f_{2}$ and $f_{4}$ can be derived from equations (5) and (6):

$x_{2}^{\prime}=r^{\prime \prime}=\frac{1}{M_{1}^{2} l_{S}^{2} \cos ^{2} \varphi-M \Theta}\left[-C M_{1} l_{S} \varphi^{\prime} \cos \varphi+\right.$

$\left.+M_{1}^{2} l_{S}^{2} g \sin \varphi \cos \varphi+\Theta F_{r} r^{\prime}-\Theta M_{1} l_{S}\left(\varphi^{\prime}\right)^{2} \sin \varphi-\Theta F\right]$

$x_{4}^{\prime}=\varphi^{\prime \prime}=\frac{1}{M_{1}^{2} l_{S}^{2} \cos ^{2} \varphi-M \Theta}\left[M C \varphi^{\prime}-M M_{1} l_{S} g \sin \varphi-\right.$

$\left.-M_{1} l_{S} F_{r} r^{\prime} \cos \varphi+M_{1}^{2} l_{S}^{2}\left(\varphi^{\prime}\right)^{2} \sin \varphi \cos \varphi+M_{1} l_{S} F \cos \varphi\right]$

Outputs of the system are identical to its states $r$ and $\varphi$ and thus their computation is straightforward.

$$
\boldsymbol{y}=\left[\begin{array}{l}
y_{1} \\
y_{2}
\end{array}\right]==\left[\begin{array}{l}
r \\
\varphi
\end{array}\right]=\left[\begin{array}{l}
x_{1} \\
x_{3}
\end{array}\right]
$$

\section{Model linearization}

Most control design techniques are based on linear model of the controlled system. If the mathematical model of the system is nonlinear, it has to be linearized around some appropriate working point (Antsaklis and Michel, 2005). In case of availability of mathematical model of the system, linearization is performed by calculating first two members of corresponding Tylor series (function value and first derivation) .

The state space linear was calculated for working point of unstable steady state (i.e. upright position of the pendulum)

$$
\boldsymbol{x}_{0}=\left[\begin{array}{c}
r_{0} \\
r_{0}^{\prime} \\
\varphi_{0} \\
\varphi_{0}^{\prime}
\end{array}\right]=\left[\begin{array}{l}
x_{10} \\
x_{20} \\
x_{30} \\
x_{40}
\end{array}\right]=\left[\begin{array}{l}
0 \\
0 \\
0 \\
0
\end{array}\right]
$$

Linearized continuous model of the inverted pendulum is calculated according following equation: 


$$
\boldsymbol{X}^{\prime}=\boldsymbol{f}(\boldsymbol{x}, 0)+\left.\frac{\partial \boldsymbol{f}}{\partial \boldsymbol{x}}\right|_{X=0, F=0} \cdot \boldsymbol{X}+\left.\frac{\partial \boldsymbol{f}}{\partial F}\right|_{X=0, F=0} \cdot F=\boldsymbol{A} \boldsymbol{X}+\boldsymbol{B} F
$$

Computation of individual elements of matrixes $\boldsymbol{A}$ and $\boldsymbol{B}$ is straightforward using equations (8)-(10).

$$
\boldsymbol{A}=\left[\begin{array}{cccc}
0 & 1 & 0 & 0 \\
0 & a_{22} & a_{23} & a_{24} \\
0 & 0 & 0 & 1 \\
0 & a_{42} & a_{43} & a_{44}
\end{array}\right], \quad \boldsymbol{B}=\left[\begin{array}{c}
0 \\
b_{2} \\
0 \\
b_{4}
\end{array}\right]
$$

where

$$
\begin{array}{lll}
a_{22}=\frac{\Theta F_{r}}{R}, & a_{23}=\frac{M_{1}^{2} l_{S}^{2} g}{R}, & a_{24}=\frac{-C M_{1} l_{S}}{R} \\
a_{42}=\frac{-M_{1} l_{S} F_{r}}{R}, & a_{43}=\frac{-M M_{1} l_{S} g}{R}, & a_{44}=\frac{C M}{R} \\
b_{2}=\frac{-\Theta}{R}, & b_{4}=\frac{M_{1} l_{S}}{R}, & R=M_{1}^{2} l_{S}^{2}-M \Theta
\end{array}
$$

The continuous linear model can be used either directly for controller design or can be transformed to its discrete equivalent with respect to sample time used in discrete controller. The second approach was used in design of MPC controller.

\section{IDENTIFICATION OF MODEL PARAMETERS}

Initial estimates of model parameters were obtained from the manufacturer of the system (Amira, 2000). This setting of parameters is summarized in table 1 .

Table 1: Initial setting of model parameters

\begin{tabular}{|l|l|l|}
\hline parameter & value & unit \\
\hline$M_{0}$ & 4.0 & $\mathrm{~kg}$ \\
\hline$M_{1}$ & 0.36 & $\mathrm{~kg}$ \\
\hline$l_{S}$ & 0.451 & $\mathrm{~m}$ \\
\hline$\Theta$ & 0.08433 & $\mathrm{~kg} \mathrm{~m}^{2}$ \\
\hline$F_{r}$ & 10.0 & $\mathrm{~kg} \mathrm{~s}^{-1}$ \\
\hline$C$ & 0.00145 & $\mathrm{~kg} \mathrm{~m}^{2} \mathrm{~s}^{-1}$ \\
\hline$k_{A}$ & 2.25 & $\mathrm{~N} / \mathrm{V}$ \\
\hline
\end{tabular}

Constant $k_{A}$ represents the gain of the servo amplifier (see figure 3 ).

Validation of this setting was performed especially by comparison of impulse responses of the real-time system and corresponding impulse responses of the Simulink model. Impulses of duration $0.3 \mathrm{~s}$ and various gain were applied to the PS600 system and courses of outputs were recorded and compared to responses obtained by Simulink model.

At first attention was paid to the cart position. The comparison of responses obtained by applying two different impulses is shown in figure 6. It can be seen that the Simulink model does not represent the real-time plant well. In case of small gain of impulse applied to the Simulink model, the final position of the cart is smaller, while greater impulses lead to smaller position comparing to the positions obtained by real PS600 system.
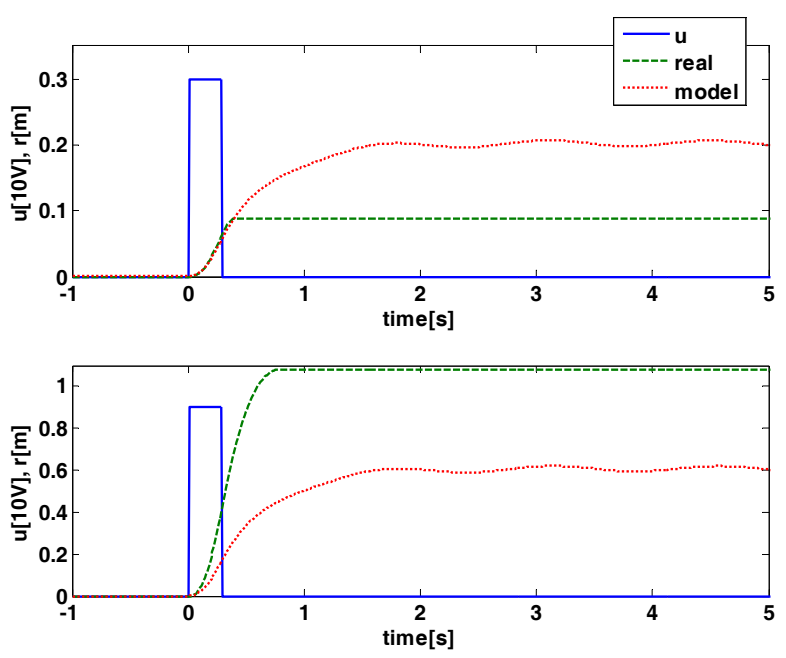

Figure 6: Comparison of impulse responses of PS600 system and its Simulink model (cart position)

By further investigation and comparison of responses, it was found out that given friction constant $F_{r}$ and servo amplifier gain $k_{A}$ do not correspond to PS600 and should be changed.

But even changes of other model parameters cannot cover the courses after the end of impulse. The slowing down of the cart is quantitatively different for model and PS600 system. The comparison of cart speed is shown in figure 7.

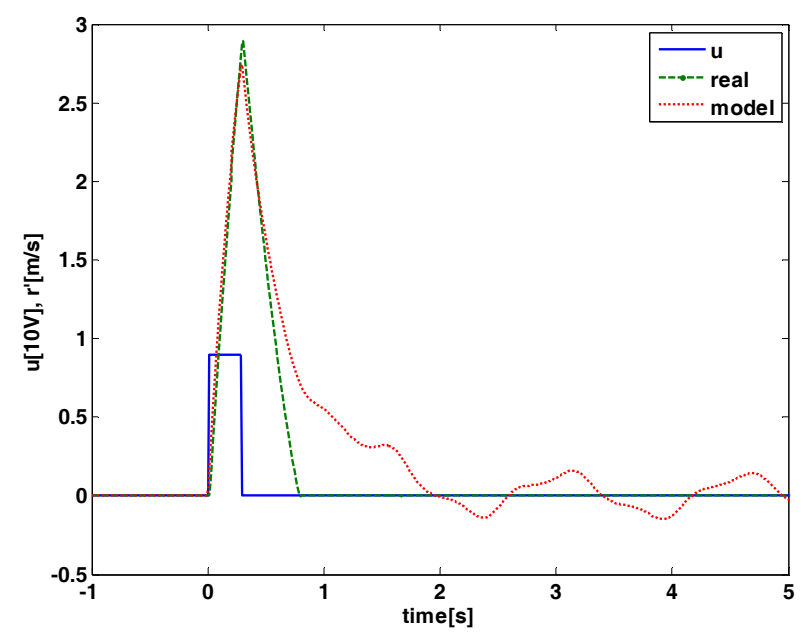

Figure 7: Comparison of impulse responses of PS600 system and its Simulink model (cart speed)

It can be seen that cart of real PS600 slows down and stops after the end of the impulse while oscillations occur in the Simulink model. These oscillations are 
caused by swinging of the pendulum and cannot be suppressed by any parameter of the Simulink model.

Non-presence of oscillations in real PS600 is caused by presence of dry (Coulomb) friction between cart and guiding bar. A force invoked by this friction acts against the movement of the cart and can be described by the following equation:

$$
F_{r C}=-F_{C} \cdot \operatorname{sign}\left(r^{\prime}\right)
$$

where $F_{C}$ is a Coulomb friction constant. The Simulink model had to be updated to cover this friction force. Model update is quite simple because the Coulomb friction acts in a similar manner as force proportional friction. The force proportional term in equations (3), (5), (9) and (10) was superseded as follows:

$$
F_{r} r^{\prime} \rightarrow F_{r} r^{\prime}+F_{c} \operatorname{sign}\left(r^{\prime}\right)
$$

Investigation of the pendulum rod angle $\varphi$ was performed in the similar manner as investigation of the cart and led also to update of model parameters. It was found out that the value of the pendulum friction constant $C$ should be updated. Moreover, the distance between centre of gravity of the pendulum and the centre of rotation $\left(l_{S}\right)$ is slightly smaller then the value given by manufacturer.

Updated model parameters of PS600 are summarized in table 2. Changed values are marked by shaded background.

Table 2: Initial setting of model parameters

\begin{tabular}{|l|l|l|l|}
\hline Parameter & $\begin{array}{c}\text { Original } \\
\text { value }\end{array}$ & New value & \multicolumn{1}{|c|}{ Unit } \\
\hline$M_{0}$ & 4.0 & 4.0 & $\mathrm{~kg}$ \\
\hline$M_{1}$ & 0.36 & 0.36 & $\mathrm{~kg}$ \\
\hline$l_{S}$ & 0.451 & 0.420 & $\mathrm{~m}$ \\
\hline$\Theta$ & 0.08433 & 0.08433 & $\mathrm{~kg} \mathrm{~m}^{2}$ \\
\hline$F_{r}$ & 10.0 & 6.5 & $\mathrm{~kg} \mathrm{~s}^{-1}$ \\
\hline$C$ & 0.00145 & 0.00652 & $\mathrm{~kg} \mathrm{~m}^{2} \mathrm{~s}^{-1}$ \\
\hline$k_{A}$ & 2.25 & 7.50 & $\mathrm{~N} / \mathrm{V}$ \\
\hline$F_{C}$ & & 15 & $\mathrm{~N}$ \\
\hline
\end{tabular}

Due to algebraic loop in the Simulink model and further nonlinearity brought by Coulomb friction, the modelling of the system by terms of Simulink blocks is not suitable. Moreover discontinuity in the Coulomb friction around $r^{\prime}=0$ leads to algebraic problems during numeric integration. The PS600 model was created in the form of Simulink s-function to cope with these problems.

Comparison of impulse responses of the PS600 and its improved model is shown in figure 8 . It can be seen that courses of signals obtained by improved model are far closer to real PS600 comparing to initial model (see figure 6). Note that pendulum angle of around $\pi$ corresponds to stable position of pendulum (i.e. pendulum rod pointing down).

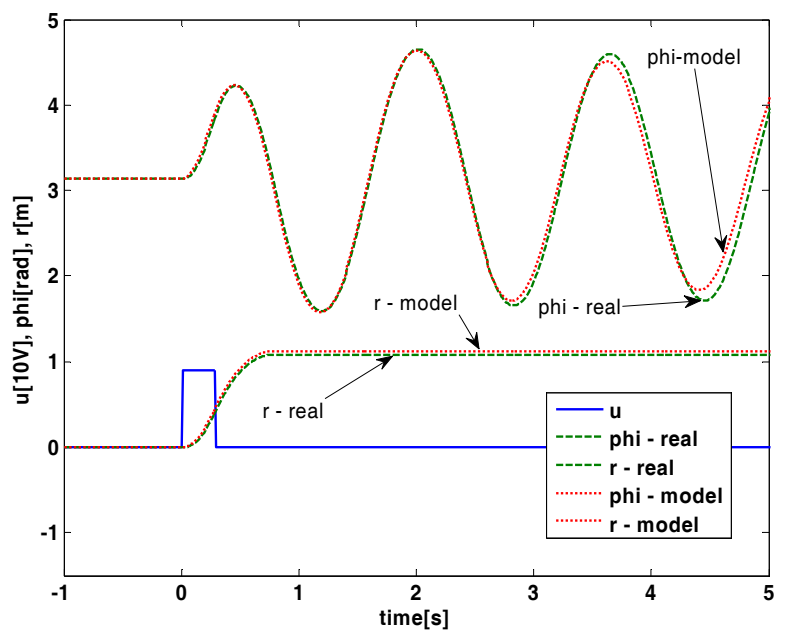

Figure 8: Comparison of impulse responses of PS600 system and its improved Simulink model (cart position and pendulum angle)

\section{MODEL PREDICTIVE CONTROL OF THE PS600}

The design of mode predictive controller for PS600 system is based on the mathematical model of the system and its linearized version. Control objectives are given as follows:

1. hold the pendulum rod in the upright position $\left(\varphi=0\right.$, i.e. $\left.w_{2}(t)=0\right)$

2. move the cart according to predefined reference trajectory $\left(r=w_{l}(t)=w(t)\right)$

The PS600 can be considered as single input two outputs system (SITO). The second output of the system $(\varphi)$ acts as a constrained because it should be close to zero during whole control process.

Generally, the computation of control signal of model predictive controller (MPC) is based on minimization of particular criterion (Kwon and Han, 2005). Usually a quadratic criterion is used (Sunan et al., 2002).:

$$
J(k)=\boldsymbol{e}^{T} \boldsymbol{Q}(k) \boldsymbol{e}+\boldsymbol{u}^{T} \boldsymbol{R}(k) \boldsymbol{u}
$$

where $\boldsymbol{e}$ is a vector of predicted control errors, $\boldsymbol{u}$ is a vector of future control signal samples and square matrixes $\boldsymbol{Q}$ and $\boldsymbol{R}$ allows to set weighting of individual vector elements. In most cases control sample differences $\boldsymbol{\Delta} \boldsymbol{u}$ are taken into account instead of $\boldsymbol{u}$, but due to integrative behaviour of the cart system the simple criterion (17) is suitable for PS600. Control sequence is obtained by minimizing criterion (17). The receding horizon is usually used: only a finite number of future values is used in the criterion and only the first element of the obtained control sequence is applied to the controlled system. 
Future outputs of the controlled system, and consequently control errors, are computed on base of the linear model. If the system is described by state space model, future system outputs depend only on current states of the system and future control signals:

$$
\boldsymbol{y}=\boldsymbol{P} \boldsymbol{x}(k)+\boldsymbol{H u}
$$

where $\boldsymbol{P}$ and $\boldsymbol{H}$ are constant matrixes. For SITO system, the vectors $\boldsymbol{y}$ and $\boldsymbol{u}$ have following form:

$$
\boldsymbol{y}=\left[\begin{array}{c}
y_{1}(k+1) \\
y_{2}(k+1) \\
y_{1}(k+2) \\
y_{2}(k+2) \\
\vdots \\
y_{1}(k+N) \\
y_{2}(k+N)
\end{array}\right], \boldsymbol{u}=\left[\begin{array}{c}
u(k) \\
u(k+1) \\
\vdots \\
u(k+N)
\end{array}\right]
$$

Proposed predictive controller simplifies criterion (17) by assigning the same weight to all samples of the same signal - matrixes $\boldsymbol{Q}$ and $\boldsymbol{R}$ are diagonal. Then the predictive control criterion can be rewritten into the following form:

$$
\begin{aligned}
J(k) & =\lambda_{1} \sum_{j=1}^{N}\left[e_{1}(k+j)\right]^{2}+\lambda_{2} \sum_{j=1}^{N}\left[e_{2}(k+j)\right]^{2} \\
& +\lambda_{3} \sum_{j=1}^{N}[u(k+j-1)]^{2}
\end{aligned}
$$

where

$$
\begin{aligned}
& e_{1}(i)=w_{1}(k+j)-y_{1}(k+j)=w_{r}(k+j)-r(k+j) \\
& e_{2}(i)=w_{2}(k+j)-y_{2}(k+j)=-\varphi(i) \\
& u(i)=F(i)
\end{aligned}
$$

where $N$ is prediction (control) horizon and $\lambda_{1}, \lambda_{2}$ and $\lambda_{3}$ are weights. Process of minimizing of the criterion can be rewritten to a quadratic programming problem:

$$
J(k)=\boldsymbol{u}^{\boldsymbol{T}} \boldsymbol{M}(k) \boldsymbol{u}+\boldsymbol{g}(k) \boldsymbol{u}
$$

where $\boldsymbol{M}$ and $\boldsymbol{g}$ are matrix and vector derived from weights and model parameters. Quadratic programming problem is usually solved numerically. This allows further constraints to be applied to vector $\boldsymbol{u}$.

\section{Simulation and real time experiments}

This section contains several results of control of the PS600 system. A Simulink control circuit used for both simulations and real time measurements is shown in figure 9. The circuit contains block for compensation of Coulomb friction. The fiction $F_{C}=15 \mathrm{~N}$ corresponds to input signal of $2 \mathrm{~V}$ (see table 2). Therefore control signal is always outside the interval of $(-2 \mathrm{~V}, 2 \mathrm{~V})$ except special case of $u=0 \mathrm{~V}$ which occurs at the beginning of control courses.

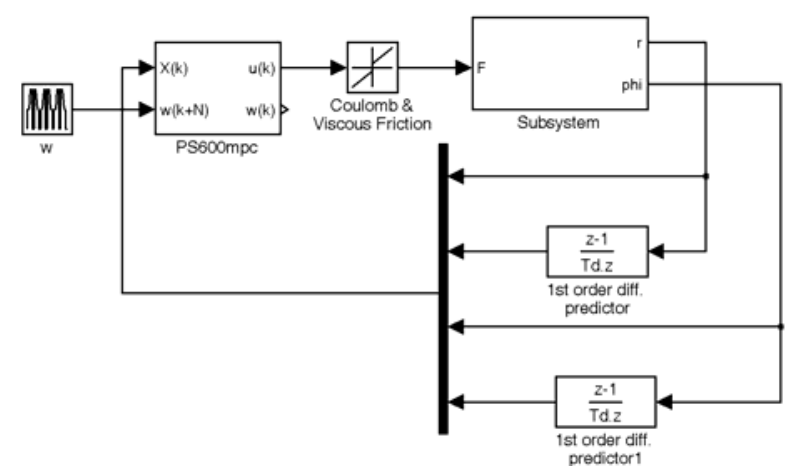

Figure 9: Simulink control circuit used for simulation and real time experiments

Precise knowledge of current states of the control system $(\boldsymbol{X}(k))$ is crucial for the MPC controller. Especially is case of unstable controlled system as PS600. Different approaches of reconstruction of immeasurable states exist. A very simple state reconstruction method is used in the scheme in figure 9. Two states are measured directly as system outputs $(r, \varphi)$ and their derivatives are to be reconstructed. A two point difference is used to compute derivatives as shown in figure 9.

First control courses were obtained by simulation and are presented in figure 10. Prediction horizon of $N=25$ samples and sample time of $T_{0}=0.04 \mathrm{~s}$ were used. Weights were set to $\lambda_{1}=10, \lambda_{2}=1$ and $\lambda_{3}=0.001$.
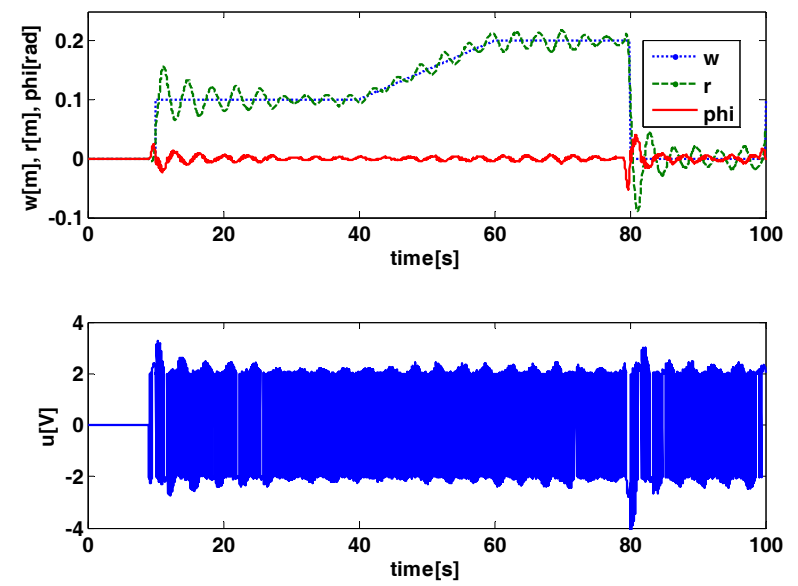

Figure 10: Simulation results of predictive control of PS600

Control of real plant with the same settings is depicted in figure 11. It can be seen that reference tracking is slightly worse than in case of figure 10 . 

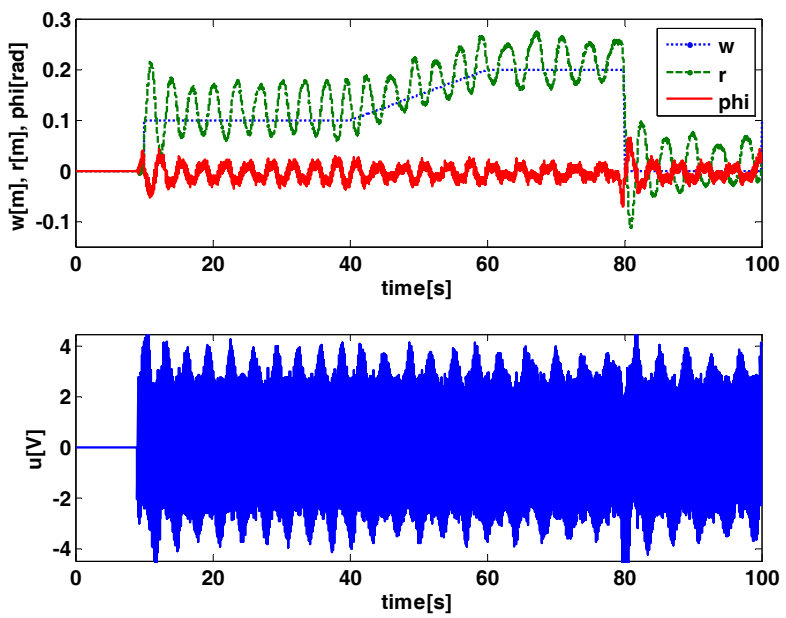

Figure 11: Real time control of PS600

An affect of different setting of weight of cart position error is shown in figure 12. In this case, all settings remained the same as in previous cases except $\lambda_{1}=50$. It is obvious that higher value of $\lambda_{1}$ leads to better reference tracking of cart position but it can be observed that pendulum angle is also smaller than in the previous case.
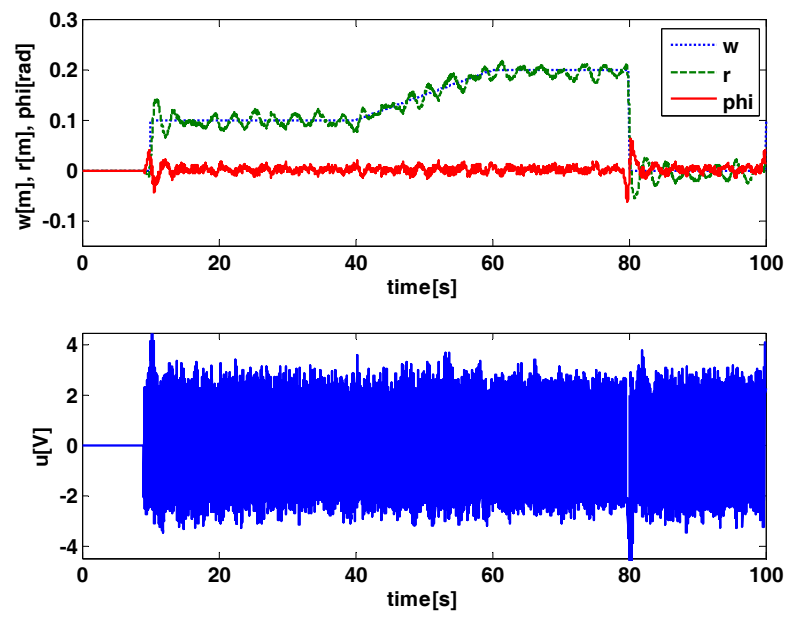

Figure 12: Real time control of PS600 $\left(\lambda_{1}=50\right)$

\section{CONCLUSION}

A mathematical mode of PS600 inverted pendulum was derived and its linearized version was calculated. Model parameters were investigated and their values were refined to obtain better correspondence between model and real equipment. Coulomb (dry) friction was also included into the model.

A model predictive controller was designed on base of a linearized discrete model. The controller was verified in both simulations and real time experiments.

Future work is to be focused especially to improvement reconstruction of immeasurable states.

\section{ACKNOWLEDGEMENTS}

This work was supported in part by the Ministry of Education of the Czech Republic under grant MSM 1M0567, and in part by the Grant Agency of the Czech Republic under grant No. 102/06/P286.

\section{REFERENCES}

Amira. 2000. PS600 Laboratory Experiment Inverted Pendulum. Amira GmbH, Duisburg.

Antsaklis, P. J. and A. N. Michel. 2005. Linear Systems. Birkhauser, Boston.

Bobál, V.; J. Böhm; J. Fessl and J. Macháček. 2005. Digital Self-tuning Controllers: Algorithms, Implementation and Applications. Springer - Verlag London Ltd., London.

Camacho, E. F. and C. Bordons. 2004. Model Predictive Control. Springer-Verlag, London.

Kwon, W. H. and S. Han (2005). Receding Horizon Control. London: Springer-Verlag.

Liu, G. P. 2001. Nonlinear identification and control - A Neural Network Approach. Springer - Verlag London Ltd., London.

Sunan, H., T. K. Kiong and L. T. Heng (2002). Applied Predictive Control. London: Springer-Verlag.

\section{AUTHOR BIOGRAPHIES}

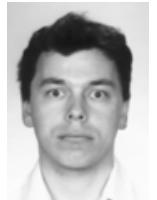

PETR CHALUPA was born in Zlin, Czech Republic in 1976. He graduated from Brno University of Technology in 1999. He obtained his Ph.D. in Technical Cybernetics at Tomas Bata University in Zlin in 2003. Nowadays he works as a researcher at Centre of Applied Cybernetics at Tomas Bata University in Zlin. His research interests are adaptive and predictive control of real-time systems. You can contact him on email address chalupa@fai.utb.cz

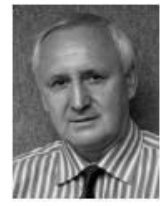

VLADIMÍR BOBÁL was born in Slavičín, Czech Republic in 1942. He graduated in 1966 from the Brno University of Technology. He received his Ph.D. degree in Technical Cybernetics at Institute of Technical Cybernetics, Slovak Academy of Sciences, Bratislava, Slovak Republic. He is now Professor in the Department of Process Control, Faculty of Applied Informatics of the Tomas Bata University in Zlín. His research interests are adaptive control systems, system identification and CAD for self-tuning controllers. You can contact him on email address bobalefai.utb.cz 